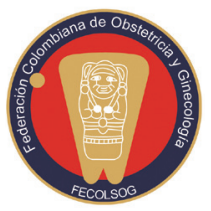

\title{
ASOCIACIÓN ENTRE LA ALTERACIÓN DEL METABOLISMO DE LA HOMOCISTEÍNA- METIONINA Y FOLATO, Y EL RETARDO DE CRECIMIENTO INTRAUTERINO IDIOPÁTICO (RCIU). DATOS PRELIMINARES
}

\section{The association between homocysteine-methionine and folate metabolism and pregnancy complicated by idiopathic intrauterine growth restriction - Preliminary data}

\author{
Reggie García-Robles, MD, PhD (c) ${ }^{1}$; Catalina Durán-Garzón ${ }^{1}$; Paola Ayala-Ramírez, \\ Bac, MSc'; Joan Dayanna Pardo-Sabogal, ND³; Rodolfo Martínez-Díaz, $\mathrm{MD}^{4}$; \\ Martha Bermúdez de Rincón, Biol, $\mathrm{PhD}^{3}$; Jaime Bernal-Villegas, $\mathrm{MD}, \mathrm{PhD}^{3}$
}

Recibido: abril 13/12 - Aceptado: diciembre 13/12

\section{RESUMEN}

Objetivo: el objetivo general de esta investigación es hacer una aproximación para evaluar la asociación entre alteración del metabolismo de la homocisteína-metionina y folato en embarazos complicados con restricción de crecimiento intrauterino (RCIU) idiopático, a través del estudio de los niveles de homocisteína pre y posparto, vitamina B12 y folato, así como las frecuencias alélicas y genotípicas de los polimorfismos genéticos de enzimas que participan en la vía metabólica de la homocisteína-metionina

1 Candidato a Doctor en Ciencias Biológicas, Instituto de Genética Humana, Pontificia Universidad Javeriana. Bogotá, Colombia. rgarcia@javeriana.edu.co

2 Estudiante de Biología, Instituto de Genética Humana, Pontificia Universidad Javeriana. Bogotá, Colombia.

3 Docente e Investigador, Instituto de Genética Humana, Pontificia Universidad Javeriana. Bogotá, Colombia.

4 Docente e Investigador, Instituto de Investigación en Nutrición, Genética y Metabolismo, Universidad El Bosque. Bogotá, Colombia.

5 Ginecoobstetra del grupo de Medicina Materno-Fetal, Departamento de Ginecología y Obstetricia, Hospital Universitario San Ignacio. Bogotá, Colombia. y folato en gestantes con embarazos complicados con RCIU idiopático y en condiciones fisiológicas en embarazo normal.

Materiales y métodos: estudio piloto observacional analítico de tipo casos y controles. Se estudiaron 8 gestantes con embarazos con restricción de crecimiento intrauterino idiopático y 21 gestantes control con embarazos sin complicaciones y recién nacido sano. Se analizaron las concentraciones de folato, vitamina B12 y homocisteína en el tercer trimestre de embarazo, así como los niveles de homocisteína posparto. Se determinaron los genotipos de las gestantes para los polimorfismos MTHFR C677T, MTHFR A1298C, MTRR A66G, CBS 844ins68, CBS VNTR31pb, CBS C699T y CBS C1080T por medio de reacción en cadena de la polimerasa (PCR) y polimorfismos de longitud de los fragmentos de restricción (RFLP).

Resultados: los polimorfismos MTHFR C677T y el genotipo 18/18 del polimorfismo CBS VNTR$31 \mathrm{pb}$, de manera independiente y en coexistencia, 
se asociaron a niveles bajos de folato. Igualmente, el genotipo 18/18 del polimorfismo CBS VNTR$31 \mathrm{pb}$, solo y cuando coexistía con el polimorfismo MTHFR C677T, se asoció con RCIU idiopático.

Conclusiones: el metabolismo de la homocisteínametionina y folato es importante para el crecimiento y desarrollo del embrión-feto. El compromiso de estas rutas metabólicas se asocia a complicaciones del embarazo como RCIU idiopático. Los resultados preliminares de este estudio deben incentivar el estudio del metabolismo de la homocisteínametionina y folato en complicaciones del embarazo como la RCIU, así como en condiciones fisiológicas en el embarazo normal en nuestra población.

Palabras clave: homocisteína, metionina, folato, restricción de crecimiento intrauterino.

\section{ABSTRACT}

Objective: This work was aimed at evaluating the association between homocysteine-metionine and folate metabolism alteration and pregnancies complicated by idiopathic intrauterine growth restriction (IUGR). This was done by studying pre- and post-partum homocysteine, vitamin B12 and folate levels, as well as allele and genotype frequency for genetic polymorphisms from enzymes participating in the homocysteine-methionine and folate metabolic route in pregnant women having pregnancies complicated by idiopathic IUGR and physiological conditions during normal pregnancy. Materials and methods: This was an analytical observational (cases and controls) pilot study. Eight pregnant women suffering IUGR were studied, as well as 21 pregnant women as control whose pregnancy had no complications and healthy newborn. Folate, vitamin and B12 homocysteine concentrations as well as postpartum homocysteine levels were analyzed during the third trimester of pregnancy. The pregnant women's genotypes were determined formethylenetetrahydrofolate reductase (MTHFR) C677T, MTHFR A1298C, methionine synthase reductase (MTRR) A66G, cystathionine betasynthase (CBS) 844ins68, CBS 31pb VNTR,
CBS C699T and CBS C1080T polymorphisms by polymerase chain reaction (PCR) and restriction fragment length polymorphism (RFLP).

Results: MTHFR C677T polymorphisms and the CBS 31bp VNTR 18/18 genotype (independently and in coexistence) were associated with low folate levels. The CBS 31bp VNTR 18/18 genotype alone and when coexisting with MTHFR C677T was associated with idiopathic IUGR.

Conclusions: Homocysteine-methionine and folate metabolism is important for embryo-fetus growth and development. Compromise of these metabolic routes is associated with pregnancy complications such as idiopathic IUGR. This study's preliminary results should encourage studying homocysteine-methionine and folate metabolism in complications regarding pregnancy such as IUGR and in physiological conditions during normal pregnancy in our population.

Key words: Homocysteine, methionine, folate, intrauterine growth restriction.

\section{INTRODUCCIÓN}

La restricción de crecimiento intrauterino (RCIU) es el cuadro clínico en el cual el feto no alcanza su pleno potencial de crecimiento, y como desenlace final se encuentra una disminución en el peso corporal, estimado prenatalmente, menor al percentil 10 para la edad gestacional y sexo según las tablas de crecimiento $(1,2)$. Es considerada una patología multifactorial, con un importante componente genético, de la cual se desconocen muchos de los mecanismos fisiopatológicos involucrados, principalmente de aquella denominada idiopática, que incluye el 40-50\% de los casos (1, 3-7). La prevalencia de RCIU varía ampliamente en los países en desarrollo, desde 10 hasta 55\%, y según lo reportado por el estudio colaborativo de antropometría materna y desenlace del embarazo de la OMS es del 17\% en Colombia (8). Tiene una importante carga en la morbilidad y mortalidad perinatal (9), además del impacto en la salud a mediano y a largo plazo (8-13). 
La homocisteína es un aminoácido sulfurado no proteico, intermediario en el metabolismo de la metionina. La metionina es un aminoácido esencial, precursor y componente de péptidos y proteínas provenientes de la dieta. El metabolismo de la homocisteína-metionina tiene dos rutas: transulfuración y remetilación $(14,15)$. El metabolismo de la homocisteína-metionina y folato participa en procesos como síntesis de purinas y pirimidinas (síntesis de ácidos nucleicos), síntesis de proteínas y generación de $\mathrm{S}$-adenosilmetionina (reacciones de metilación) (15-17). Las concentraciones sanguíneas de homocisteína son determinadas por factores genéticos como los polimorfismos o las variaciones en los genes de las enzimas implicadas en estas rutas metabólicas, y por factores nutricionales como los niveles de vitamina B12, folato y vitamina B6 (14).

Durante el embarazo, en condiciones normales, se incrementan los requerimientos de folato y disminuye la concentración de homocisteína total plasmática (tHcy) en comparación con mujeres no embarazadas $(18,19)$, lo que sugiere un papel importante de esta vía metabólica durante el embarazo $(13,20)$. Se ha reportado que las concentraciones basales de tHcy disminuyen gradualmente durante el inicio y la mitad del embarazo hasta alcanzar los valores inferiores al final del mismo, sin disminución posterior $(13,20)$. Mientras que los niveles de homocisteína posparto, además de ser mayores que los del embarazo, son similares a los del periodo preconcepcional $(13,19)$. Por otra parte, aunque es claro que los niveles de vitamina B12 y folato disminuyen durante la gestación, no se sabe cuáles son los factores involucrados y el papel de cada uno de ellos. Se ha postulado que la hemodilución, la influencia hormonal, el aumento de los requerimientos y el déficit nutricional podrían ser factores importantes (21). Durante el embarazo, la alteración del metabolismo de la homocisteína-metionina y folato ha sido asociada a trombosis venosa profunda, desprendimiento prematuro de placenta, preeclampsia, defecto del tubo neural, RCIU y muerte fetal $(22,23)$.

En la revisión de la literatura evidenciamos que el metabolismo de la homocisteína-metionina y folato juega un papel importante en el desarrollo normal del embarazo, y en el crecimiento y desarrollo del embrión-feto, y es probable que la alteración de esta vía metabólica, que podría ser de origen genético, se asocie al desarrollo de RCIU. El objetivo general de esta investigación es hacer una aproximación para evaluar la asociación entre alteración del metabolismo de la homocisteína-metionina y folato con embarazos complicados con RCIU, a través del estudio de los niveles de homocisteína pre y posparto, vitamina B12 y folato, así como las frecuencias alélicas y genotípicas de los polimorfismos genéticos de enzimas que participan en la vía metabólica de la homocisteína-metionina y folato en gestantes con embarazos complicados con restricción de crecimiento intrauterino y en embarazo normal.

\section{MATERIALES Y MÉTODOS}

Estudio observacional analítico de tipo casos y controles. Se incluyeron como casos gestantes con embarazos complicados con RCIU definido como peso fetal estimado menor al percentil 10 para la edad gestacional y sexo según las tablas de crecimiento. Se usaron curvas de peso al nacer de población colombiana para evaluar el peso según la edad gestacional y el sexo del recién nacido (24). Se excluyeron de los casos las gestantes con embarazos con RCIU de causa conocida, fetos-recién nacidos con malformaciones fetales mayores o impresión diagnóstica de patología genética, gestantes con condiciones protrombóticas y mortinatos, así como evidencia de alguna de estas patologías en el análisis histopatológico. El grupo control correspondió a gestantes con embarazo sin complicaciones y con recién nacido sano, sin evidencia de alteración de la placenta en el análisis histopatológico. Las gestantes evaluadas fueron reclutadas para el estudio en el periodo comprendido entre septiembre de 2009 y diciembre de 2010 a través del servicio de Ginecología y Obstetricia del Hospital Universitario San Ignacio (IV nivel de complejidad) de Bogotá que atiende pacientes del aseguramiento contributivo. 
Tamaño muestral y muestreo: se reclutaron 8 casos y 21 controles, a través de muestreo por conveniencia.

Procedimiento: previa autorización para ingresar al estudio mediante la firma del consentimiento informado se diligenció formato para recolección de datos relevantes de la historia clínica, cuestionario de hábitos alimentarios y recordatorio 24 horas de la madre para evaluar algunas variables de interés; se realizó examen físico y valoración nutricional. El cuestionario de hábitos alimentarios y el recordatorio 24 horas son los usados en la valoración de rutina durante la consulta nutricional. Además, a las gestantes se les tomó una muestra de sangre en dos tubos (5 cc) con EDTA para el análisis de los polimorfismos genéticos y en un tubo con heparina $(5$ cc) para cuantificación de homocisteína (durante el tercer trimestre de embarazo y tres meses posparto), folato y vitamina B12 en plasma, en ayuno. Las muestras fueron almacenadas a $4{ }^{\circ} \mathrm{C}$ hasta su procesamiento. Los niveles plasmáticos de folato y vitamina B12 se determinaron mediante un inmunoensayo enzimático quimioluminiscente competitivo (25). La concentración de tHcy se determinó por inmunoensayo competitivo basado en inmunoanálisis de polarización de la fluorescencia (fluorescence polarization immunoassay, FPIA) (26). El ADN genómico se aisló a partir de sangre periférica mediante el método Salting out o "extrac- ción salina" (27). Las condiciones de la amplificación para el análisis del polimorfismo C677T de la MTHFR y de los polimorfismos C699T, C1080T y 844ins68 de la CBS fueron descritas por Bermúdez et al. (28). Para los polimorfismos CBS C699T, CBS C1080T, MTHFR C677T, MTHFR A1298C (29) y MTRR A66G (30) se realizó amplificación del fragmento de interés por reacción en cadena de la polimerasa (PCR) y genotipificación por polimorfismos de longitud de los fragmentos de restricción (RFLP). Para el análisis del polimorfismo 844ins68 del gen CBS se realizó amplificación de un fragmento del intrón 7 - exón 8, de 805 pares de bases para el "wild type", y de 873 pares de bases cuando el polimorfismo está presente. Para el análisis del polimorfismo VNTR 31pb del gen CBS se realizó amplificación por PCR de fragmentos entre 660908 pares de bases y se genotipificó de acuerdo con la longitud del fragmento por la presencia de los polimorfismos de 13, 15, 16, 17, 18, 19, 20 y 21 repeticiones (31-34) (tabla 1). Esta metodología fue revisada y analizada con las herramientas electrónicas: Primer3 (35) y Rebase (36).

Variables medidas: edad materna, edad gestacional (tomada de la historia clínica y determinada por el método de Ballard), talla materna, peso pregestacional, índice de masa coproral (IMC) pregestacional, incremento de peso en el embarazo, periodo inter-

\begin{tabular}{|c|c|c|c|}
\hline \multicolumn{4}{|c|}{$\begin{array}{c}\text { Tabla } 1 . \\
\text { Condiciones para la genotipificación de los polimorfismos genéticos }\end{array}$} \\
\hline Polimorfismo & Primer con sentido & Primer antisentido & $\begin{array}{c}\text { Enzima } \\
\text { de } \\
\text { restricción }\end{array}$ \\
\hline CBS C699T & 5'CAGCAACCCCCTGGCTCACT3' & 5'CAGCCATGCCCTGTGTTTGCTATT3' & RsaI \\
\hline CBS C1080T & 5'CAGTGCCCACCCCAGCTCATTA3' & 5'GGCCTCCTCCCCTCCCAGTTCT3' & BstUI \\
\hline MTHFR C677T & 5'TGAAGGAGAAGGTGTCTGCGGGA3' & 5'AGGACGGTGCGGTGAGAG3' & Taq I \\
\hline MTHFR A1298C & 5'ATGTGGGGGGAGGAGCTGAC3' & 5' 'GTCTCCСАACTTACCCTTCTCСС 3 ' & MboII \\
\hline MTRR A66G & 5'AGCAGGGACATGCAAAGGCCATCGCAGAAGACAT3' & 5'CTCTAACCTTATCGGATACACTAATA3' & NspI \\
\hline CBS 844ins68 & 5'CCGCAGGGTGGTCTGTCTGGACTG3' & 5'AGCCCCACTGAGCATCCGTGTGAC3' & No Aplica \\
\hline CBS VNTR3 $1 p b$ & 5'TGCAGCCGTCAGACCAAG3' & 5'TTAAGTCCCCAAAACACGG3' & No Aplica \\
\hline
\end{tabular}


genésico, primigravidez, antecedente de RCIU, condiciones socioeconómicas desfavorables (gestantes pertenecientes a estratos 1-3), consumo habitual de frutas y verduras, ejercicio regular periconcepcional, ingesta de multivitamínicos, ingesta de ácido fólico, de sulfato ferroso, y de calcio, peso, sexo y talla del recién nacido, niveles de homocisteína en el embarazo $(\mu \mathrm{mol} / \mathrm{L})$, niveles de homocisteína posparto $(\mu \mathrm{mol} / \mathrm{L})$, cambio en los niveles de homocisteína posparto respecto a los gestacionales (presentado como el porcentaje de disminución de la concentración en el embarazo con respecto a la concentración de homocisteína plasmática posparto), hiperhomocisteinemia (definida con valores $>$ 9,16 $\mathrm{mol} / \mathrm{L}(\bar{X}:(5,47)+2 \mathrm{DE}:(1,84))$ de acuerdo con nuestros resultados, $\mathrm{y}>10,5 \mu \mathrm{mol} / \mathrm{L}$ de acuerdo con López-Quesada et al.) (37), concentración de folato plasmático $(\mathrm{ng} / \mathrm{mL})$, déficit moderado de folato (3,0-6,0 ng/mL) según González-Gross et al. (38), déficit de vitamina B12 $(\leq 200 \mathrm{pg} / \mathrm{mL})$ de acuerdo con Stabler et al. (39). También se determinaron los alelos y los genotipos para los polimorfismos CBS VNTR 31pb, CBS 844ins68, CBS C1080T, CBS C699T, MTRR A66G, MTHFR C677T y MTHFR A1298C.

Análisis: las variables cuantitativas fueron descritas como mediana y rango. Las variables categóricas se presentaron con frecuencias absolutas y relativas. Se realizó análisis con prueba exacta binomial para la comparación de las frecuencias, cuando el evento tuvo frecuencia de cero se atribuyó la presencia de un evento entre todos los sucesos evaluados (como menor probabilidad posible) para poder hacer análisis con prueba binomial exacta. Se utilizó la prueba de Mann Whitney para la comparación de las medianas. Las pruebas estadísticas se realizaron con el programa Stata versión 9.1. Las frecuencias alélicas y genotípicas se calcularon por conteo manual. Se realizó la evaluación del equilibrio HardyWeinberg en el grupo control con el programa Genepop versión 4.0.

Aspectos éticos: se contó con el aval del comité de ética de la Facultad de Medicina de la Pontificia
Universidad Javeriana. El ingreso de cada paciente al estudio se hizo previo consentimiento informado.

\section{RESULTADOS}

Las características basales y los antecedentes nutricionales fueron similares entre los casos y los controles. A pesar de que los casos tenían una mediana $7 \mathrm{~kg}$ menor que los controles, esta diferencia no se consideró clínicamente significativa debido a que el grupo control tenía una talla ligeramente mayor y no se encontraron diferencias estadísticamente significativas en el índice de masa corporal (IMC) (tabla 2). Se encontraron diferencias estadísticamente significativas en el antecedente de embarazo con RCIU y en el peso del recién nacido (tabla 3).

Ninguna de las gestantes evaluadas -ni casos ni controles- tuvo una ingesta regular y adecuada de ácido fólico, sulfato ferroso o multivitamínicos. Solo una gestante del grupo control tomo ácido fólico preconcepcional. El 62,50\% de los casos, y el $71,43 \%$ de los controles fueron clasificadas como gestantes con dieta adecuada de metionina $(\mathrm{p}=0,6964)$; por otra parte, el 50\% de los casos y el 38,10\% de los controles recibieron una dieta adecuada en folato ( $p=0,4899)$. Respecto a la vitamina B12, 50\% de los casos y 52,38\% de los controles tuvieron una dieta adecuada $(\mathrm{p}=1,0000)$.

No se encontró diferencia entre los grupos de pacientes con RCIU y el grupo control en los niveles de homocisteína durante el embarazo ni posparto, folato o vitamina B12; tampoco se observaron diferencias estadísticamente significativas entre los grupos en la disminución de la concentración de tHcy en el embarazo. Se evaluaron los niveles de tHcy posparto en 14 de los 21 controles $(66,66 \%)$ (tabla 4). En cuanto a la evaluación de hiperhomocisteinemia en el embarazo, no se encontró ninguna gestante con esta condición bajo los dos criterios utilizados. Por otro lado, 23,80\% de las gestantes control tuvieron déficit mixto de folato y vitamina B12, y 47,61\% presentaron algún tipo de déficit de estas vitaminas, solo en el 28,57\% de las gestantes control se encontraron niveles normales de estas 


\begin{tabular}{|c|c|c|c|}
\hline \multicolumn{4}{|c|}{$\begin{array}{c}\text { Tabla } 2 . \\
\text { Variables maternas en los grupos de estudio }\end{array}$} \\
\hline Variable & $\operatorname{Casos} n=8$ & Controles $\mathbf{n}=21$ & Valor de $\mathbf{P}$ \\
\hline Edad materna (años) & $26,50(20-34)$ & $28,00(20-38)$ & 0,3649 \\
\hline Talla materna $(\mathrm{cm})$ & $154,50(148-168)$ & $156,00(150-168)$ & 0,2858 \\
\hline Peso pregestacional (kg) & $49,00(39-78)$ & $56,00(49-74)$ & 0,1366 \\
\hline IMC pregestacional & $21,35(17,80-28,65)$ & $23,01(18,82-27,85)$ & 0,2025 \\
\hline Incremento de peso en el embarazo (kg) & $10,50(6,00-17,50)$ & $13,00(8,00-20,00)$ & 0,4235 \\
\hline Periodo intergenésico (meses) & $72,00(5,00-84,00)$ & $60,00(12,00-144,00)$ & 0,9373 \\
\hline Primigravidez & $3(37,50)$ & $3(14,29)$ & 0,0934 \\
\hline Antecedente de RCIU & $4(80,00)$ & $0(0,00)$ & $<0,0001$ \\
\hline Condiciones socioeconómicas desfavorables* & $6(75,00)$ & $19(95,00)$ & 0,0572 \\
\hline Consumo habitual de frutas y verduras & $5(62,50)$ & $10(47,62)$ & 0,4905 \\
\hline Ejercicio regular periconcepcional & $1(12,50)$ & $1(4,76)$ & 0,3230 \\
\hline Ingesta de multivitamínicos & $3(60,00)$ & $6(28,57)$ & 0,6964 \\
\hline Ingesta de ácido fólico & $7(87,50)$ & $14(66,67)$ & 0,2829 \\
\hline Ingesta de sulfato ferroso & $6(75,00)$ & $11(52,38)$ & 0,2935 \\
\hline Ingesta de calcio & $3(60,00)$ & $7(33,33)$ & 0,7268 \\
\hline
\end{tabular}

Las variables son presentadas con mediana (rango) y con frecuencia absoluta (\%). IMC: índice de masa corporal

\begin{tabular}{|l|c|c|c|}
\hline \multicolumn{5}{c}{ Tabla 3. } \\
\multicolumn{2}{|c|}{ Variables del recién nacido en los grupos de estudio } \\
\hline Variable & Casos $\mathbf{n}=8$ & Controles $\mathbf{n}=21$ & Valor de P \\
\hline Edad gestacional (semanas) & $37,50(31,00-40,00)$ & $38,00(37,00-42,00)$ & 0,1052 \\
\hline Peso al nacer $(\mathrm{g})$ & $2230(895-2775)$ & $2855(2470-3460)$ & 0,0003 \\
\hline Talla al nacer $(\mathrm{cm})$ & $47,50(33-51)$ & $48,00(45-52)$ & 0,2642 \\
\hline Masculino & $2(25,00)$ & $7(33,33)$ & 1,0000 \\
\hline Femenino & $6(75,00)$ & $14(66,67)$ & \\
\hline
\end{tabular}

Las variables están presentadas con mediana (rango) y con frecuenacia absoluta (\%).

vitaminas en el tercer trimestre del embarazo. Los niveles de la vitamina B12 no pudieron ser analizados cuantitativamente debido a que el resultado reportado en un caso y seis controles fue $<150 \mathrm{pg} /$ $\mathrm{mL}$, límite de lectura de la prueba utilizada.

La valoración del equilibrio de Hardy-Weinberg en los controles evidenció que las frecuencias genotípicas de los polimorfismos se encontraban en equilibrio $(\mathrm{p}>0,05)$. Se hallaron diferencias estadísticamente significativas $(p=0,0024)$ en las concentraciones de folato plasmático entre las gestantes portadoras del alelo T (genotipos TT y CT) del polimorfismo MTHFR C677T y las no portadoras (CC), 5,54 ng/mL (rango: 4,26-14,30) y 8,98 ng/ mL (rango: 6,66-19,9), respectivamente. Asimismo, se halló asociación entre el estado portador del alelo T (p = 0,0002) y los genotipos TT y CT (p < $0,0001)$ con el déficit de folato. Vale la pena resaltar que ninguna de las gestantes control con genotipo CC tuvo déficit de folato. Para el polimorfismo CBS 


\begin{tabular}{|c|c|c|c|}
\hline Variable & $\operatorname{Casos} n=8$ & Controles $n=21$ & Valor de $p$ \\
\hline Niveles homocisteína en el embarazo $(\mu \mathrm{mol} / \mathrm{L}) \S$ & $5,48(2,35-8,98)$ & $5,20(2,36-8,48)$ & 0,7403 \\
\hline Niveles de homocisteína posparto $(\mu \mathrm{mol} / \mathrm{L}) \S$ & $6,63(4,85-8,30)$ & $6,67(4,83-10,10)$ & 0,8317 \\
\hline Diferencia entre homocisteína en embarazo y posparto & $35,92(3,28-51,55)$ & $19,27(3,04-69,90)$ & 0,5954 \\
\hline Concentración de folato plasmático $(\mathrm{ng} / \mathrm{mL})^{*}$ & $8,81(4,62-18,80)$ & $6,58(4,26-19,90)$ & 0,1959 \\
\hline Déficit moderado de folato $(3,0-6,0 \mathrm{ng} / \mathrm{mL})^{* *}$ & $1(12,50)$ & $9(42,86)$ & 0,1495 \\
\hline Déficit de vitamina B12 $(\leq 200 \mathrm{pg} / \mathrm{mL})^{* *}$ & $3(37,50)$ & $11(52,38)$ & 0,4905 \\
\hline
\end{tabular}

VNTR31pb se observó que las gestantes con genotipo 18/18 tenían niveles más bajos de folato (mediana: 5,84 ng/mL; rango: 4,26-14,30) con respecto a las no portadoras de este genotipo (mediana: 7,58 ng/mL; rango: 4,50-19,90), sin diferencias estadísticamente significativas $(p=0,1688)$. Sin embargo, la presencia del alelo 18 ( $\mathrm{p}=0,0050)$ y del genotipo 18/18 ( $\mathrm{p}=$ $0,0242)$ se encontró asociada a déficit de folato. También se observó que el estado portador del genotipo 18/18 del polimorfismo CBS VNTR31pb y portador de genotipo TT o CT del polimorfismo MTHFR C677T estuvo asociado con niveles bajos de folato plasmático (mediana: 5,47 ng/mL; rango: 4,26-14,3) comparado con las gestantes no portadoras de estos genotipos (mediana: 7,50 ng/mL; rango: 4,50-19,90), con diferencias estadísticamente significativas $(\mathrm{p}=$ 0,0346). La presencia de estos genotipos también se asoció a déficit moderado de folato $(\mathrm{p}=0,0004)$, $70 \%$ déficit de folato en gestantes con genotipos 18/18 y CT o TT, y 22,22\% de gestantes con déficit de folato en las gestantes no portadoras de esta combinación de genotipos. No se encontraron otras asociaciones entre los niveles de tHcy, folato y vitamina B12 para los demás polimorfismos evaluados en el grupo control, ni se encontraron diferencias estadísticamente significativas entre los niveles de tHcy, folato y vitamina B12, así como de los estados de déficit vitamínicos, entre los grupos caso y control.

Con respecto a los polimorfismos genéticos se evidenció asociación del genotipo 18/18 del polimorfismo CBS VNTR31pb con RCIU ( $\mathrm{p}=$ 0,0277). La presencia simultánea del genotipo 18/18 del polimorfismo CBS VNTR31pb y de los genotipos TT o CT del polimorfismo MTHFR C677T también se asoció a RCIU ( $p=0,0315)$. Los demás polimorfismos genéticos evaluados no se asociaron con RCIU (tabla 5).

\section{DISCUSIÓN}

No se encontró asociación entre los niveles de homocisteína pre y posparto, vitamina B12 y folato preparto, y tampoco se observaron diferencias estadísticamente significativas entre los grupos en la disminución de la concentración de tHcy en el embarazo. Encontramos que la concentración de tHcy materna en el tercer trimestre de embarazos sin complicaciones es menor que la concentración tres meses posparto, lo cual corresponde a lo reportado en la literatura $(13,14,18,19,40)$. Sin embargo, las concentraciones durante el embarazo fueron 19,27\% menores que los niveles posparto, valor inferior a lo reportado en la literatura (29$60 \%)(19,41,42)$. Al comparar los niveles de tHcy $(5,20 \mu \mathrm{mol} / \mathrm{L})$ obtenidos durante el tercer trimestre de embarazos normales con los reportados por otros estudios, encontramos que son semejantes a lo descrito por Patrick et al. (43) en Estados Unidos (5,5 $\mu \mathrm{mol} / \mathrm{L})$ y Murphy et al. (41) en España (5,16 $\mu \mathrm{mol} / \mathrm{L}), \mathrm{y}$ menores a los encontrados por Sánchez et al. (44) en Perú y López-Quesada et al. (37) en 


\begin{tabular}{|c|c|c|c|c|}
\hline \multicolumn{2}{|c|}{ Polimorfismo } & Casos $n=8$ & Controles $n=21$ & Valor de $p$ \\
\hline \multirow[t]{2}{*}{ CBS VNTR 31pb } & Alelo 18 & $16(100,00)$ & $34(80,95)$ & 0,0546 \\
\hline & Genotipo 18/18 & $8(100,00)$ & $13(61,90)$ & 0,0277 \\
\hline \multirow[t]{2}{*}{ CBS 844ins68 } & Alelo ins & $0(0,00)$ & $2(9,52)$ & 1,0000 \\
\hline & Genotipo Wt/ins & $0(0,00)$ & $2(4,76)$ & 1,0000 \\
\hline \multirow[t]{3}{*}{ CBS C1080T } & Alelo T & $4(28,57)$ & $11(28,95)$ & 1,0000 \\
\hline & Genotipo TT & $1(14,29)$ & $3(15,79)$ & 1,0000 \\
\hline & Genotipo TT o CT & $3(42,86)$ & $8(42,11)$ & 1,0000 \\
\hline \multirow[t]{2}{*}{ CBS C699T } & Alelo T & $2(12,50)$ & $12(28,57)$ & 0,2655 \\
\hline & Genotipo CT & $2(25,00)$ & $12(57,14)$ & 0,0813 \\
\hline \multirow[t]{3}{*}{ MTRR A66G } & Alelo G & $9(56,25)$ & $16(38,10)$ & 0,1960 \\
\hline & Genotipo GG & $3(37,50)$ & $5(23,81)$ & 0,4054 \\
\hline & Genotipo GG o AG & $6(75,00)$ & $11(52,38)$ & 0,2935 \\
\hline \multirow[t]{3}{*}{ MTHFR C677T } & Alelo T & $10(62,50)$ & $18(42,86)$ & 0,1324 \\
\hline & Genotipo TT & $3(37,50)$ & $5(23,81)$ & 0,4054 \\
\hline & Genotipo TT o CT & $7(87,50)$ & $13(61,90)$ & 0,1667 \\
\hline \multirow[t]{3}{*}{ MTHFR A1298C } & Alelo C & $2(12,50)$ & $9(21,43)$ & 0,5479 \\
\hline & Genotipo CC & $0(0,00)$ & $1(4,76)$ & 1,0000 \\
\hline & Genotipo CC o AC & $2(25,00)$ & $8(38,10)$ & 0,7185 \\
\hline
\end{tabular}

Se presentan las frecuencias alélicas y genotípicas de los polimorfismos como frecuencia absoluta (\%). No se encontraron individuos con genotipo TT para el polimorfismo CBS C699T ni en las gestantes control ni en gestantes con embarazo con RCIU.

España, 6,7 $\mu \mathrm{mol} / \mathrm{L}$ y $6,3 \mu \mathrm{mol} / \mathrm{L}$, respectivamente. Llama la atención que los niveles de tHcy no sean mayores debido a la frecuencia de niveles bajos de folato y vitamina B12, aunque es posible que el déficit de estas vitaminas sea de origen nutricional y esté acompañado de baja ingesta de alimentos ricos en metionina. La baja ingesta de metionina podría influir negativamente en la concentración de tHcy. Una dieta asociada a concentraciones altas de tHcy es probablemente baja en otros nutrientes además del folato, y los resultados pueden reflejar un déficit nutricional más general. Además, aunque la concentración elevada de tHcy es un marcador sensible del estado de folato, otros nutrientes y otros factores no nutricionales están también asociados (45). Los niveles de folato plasmático en los controles mostraron que el 42,86\% de las madres cursaban con déficit moderado en el tercer trimestre de embarazo.
En relación con este hallazgo debemos considerar un consumo insuficiente de alimentos ricos en esta vitamina que permita suplir la demanda del feto (38), así como conductas que podrían disminuir el contenido de esta vitamina en los alimentos, como cocinar los vegetales (46). Vale la pena resaltar que en la literatura existe evidencia que sugiere que la suplementación con ácido fólico puede reducir la prevalencia de anemia megaloblástica, defectos del tubo neural, labio paladar hendido, preeclampsia, abruptio de placenta, RCIU y muerte fetal $(17,47$, 48), por lo cual se reglamentó la fortificación de alimentos con ácido fólico en varios países y se recomendó la suplementación con ácido fólico en otros (49-51). Al respecto, se observó que las gestantes no realizaban la suplementación de acuerdo con las recomendaciones de los expertos, situación reportada como una dificultad en los países que no tienen 
políticas de fortificación de alimentos (47). Además, en el grupo control se encontraron niveles muy bajos de vitamina $\mathrm{B} 12(\leq 150 \mathrm{pg} / \mathrm{mL})$ en 28,57\% de las madres, con una frecuencia de déficit de 52,38\%. $\mathrm{Al}$ respecto, se ha sugerido que muchas gestantes podrían tener dietas bajas en consumo de proteínas de origen animal, fuente dietética de vitamina B12, debido a sus precios elevados en el mercado (21, 52, 53). Las infecciones parasitarias o bacterianas también podrían desempeñar un papel en el déficit de vitamina B12. Igualmente, las gestantes con déficit de vitamina B12 tuvieron con frecuencia concentraciones bajas de folato, lo que parece indicar un déficit originado en la dieta. Sin embargo, concentraciones bajas de folato también podrían ser secundarias al déficit de vitamina B12, ya que ante el déficit de esta vitamina el 5-metiltetrahidrofolato es atrapado y no puede reincorporarse a la ruta metabólica del folato (21). El déficit de vitamina B12 ha empezado a ser reconocido como un problema de salud pública a nivel mundial, y el de origen nutricional ha sido reportado como un problema grave en India, México, Sur-Centro América y algunos países de África con implicaciones en la salud de la madre y el feto (21, $39,52-54)$. Por estas razones se ha sugerido que se debería realizar fortificación de alimentos con vitamina B12, con el fin de suplir el déficit en ciertos grupos demográficos como jóvenes, mujeres en edad fértil, gestantes y ancianos (55-56).

En cuanto al hallazgo positivo de asociación de antecedente de RCIU, encontramos que estos resultados están acordes con lo reportado en la literatura. Ghezzi et al. reportaron incremento de 6,7 veces más riesgo de tener un embarazo complicado con RCIU cuando existía el antecedente de esta restricción en un embarazo previo, lo que soporta el concepto de importantes factores genéticos heredables que participan en la fisiopatología de esta entidad (57).

Por otro lado, el hallazgo de asociación de la presencia del polimorfismo MTHFR C677T con niveles menores y déficit de folato ya ha sido reportado en la literatura $(45,57,58)$. También se encontró asociación de presencia del alelo 18 y genotipo 18/18 con déficit de folato. En la literatura no se encuentra descripción de esta asociación; no obstante, es probable que el compromiso de la vía de la transulfuración tenga un impacto en la vía de la remetilación. Adicionalmente, la combinación de genotipos 18/18 del polimorfismo CBS VNTR31pb y TT o CT del polimorfismo MTHFR C677T se asoció a niveles menores de folato y déficit moderado de folato plasmático. De esta relación tampoco se encontró reporte en la literatura, aunque sí había sido reportada la interacción de ambos polimorfismos con efecto aditivo sobre los niveles de tHcy (32).

Respecto a los polimorfismos genéticos se evidenció asociación del genotipo 18/18 del polimorfismo CBS VNTR31pb, así como de la presencia simultánea de los genotipos 18/18 del polimorfismo CBS VNTR 31pb y genotipo TT o CT del polimorfismo MTHFR C677T con RCIU. No se encontró reporte de estos hallazgos en la literatura. No obstante, el genotipo 18/18 se ha asociado a compromiso del metabolismo de la homocisteína-metionina y aumento de los niveles de tHcy (16). En cuanto al polimorfismo MTHFR C677T, este es responsable de la variante termolábil de la MTHFR, que produce una actividad reducida de la enzima y, como consecuencia, aumento en los niveles de tHcy (28). Además, la evidencia indica que este polimorfismo no solo se relaciona con niveles elevados de tHcy y disminuidos de folato, sino con un incremento en el riesgo de aborto recurrente, infertilidad femenina, RCIU y defectos del tubo neural $(45,58,59)$. Cabe resaltar que también se ha reportado que el polimorfismo y niveles bajos de folato tienen un efecto aditivo, y a su vez el efecto del polimorfismo puede ser modulado por niveles adecuados de folato (60).

\section{CONCLUSIONES}

Estos resultados preliminares sugieren que el metabolismo de la homocisteína-metionina y folato sufre modificaciones durante el embarazo, que existe una frecuencia relevante de déficit de folato y de vitamina B12 en las gestantes colombianas, así como la participación de polimorfismos genéticos en los niveles de 
folato y en la ocurrencia de RCIU idiopático. Finalmente, nos incentivan para continuar el estudio del metabolismo de la homocisteína-metionina y folato en el embarazo normal, así como en complicaciones del embarazo como la RCIU en Colombia.

Agradecimientos: a todo el personal del Departamento de Ginecología y Obstetricia del Hospital Universitario San Ignacio por su valiosa colaboración en la toma de las muestras.

\section{REFERENCIAS}

1. Muñoz L, Hernández R. Retardo de crecimiento intrauterino (RCIU) y sus alteraciones bioquímicas. NOVA 2005;3:88-94.

2. Vélez MP, Barros FC, Echavarría LG, Hormaza MP. Prevalencia de bajo peso al nacer y factores maternos asociados: Unidad de Atención y Protección Materno Infantil de la Clínica Universitaria Bolivariana, Medellín, Colombia. Rev Colomb Obstet Ginecol 2006;57:264-70.

3. Kaufmann P, Black S, Huppertz B. Endovascular trophoblast invasion: implications for the pathogenesis of intrauterine growth retardation and preeclampsia. Biol Reprod 2003;69:1-7.

4. Prieto R, Matamala F, Rojas M. Morphometric and Morphological Characteristics of the Placenta at Term in Small Gestational Age Newborns (SGA) in the City of Temuco-Chile. Int J Morphol 2008;26:615-21.

5. Cuartas A. Retardo del crecimiento intrauterino. Iatreia 1995;8:18-25.

6. Maulik D, Frances J, Ragolia L. Fetal growth restriction: pathogenic mechanisms. Clin Obstet Gynecol 2006;49:219-27.

7. Maulik D. Fetal growth restriction: the etiology. Clin Obstet Gynecol 2006;49:228-35.

8. Kramer MS. The Epidemiology of Adverse Pregnancy Outcomes: An Overview. J Nutr 2003;133:1592S-6s.

9. Alexander GR, Kogan M, Bader D, Carlo W, Allen M, Mor J.US birth weight/gestational age-specific neonatal mortality: 1995-1997 rates for whites, Hispanics, and blacks. Pediatrics 2003;111:e61-6.10.
Philip AG. The evolution of neonatology. Pediatr Res 2005;58:799-815.

11. Kramer MS, Séguin L, Lydon J, Goulet L. Socioeconomic disparities in pregnancy outcome: why do the poor fare so poorly? Paediatr Perinat Epidemiol 2000;14:194-210.

12. Scifres CM, Nelson DM. Intrauterine growth restriction, human placental development and trophoblast cell death. J Physiol 2009;587:3453-8.

13. Cikot RJ, Steegers-Theunissen RP, Thomas CM, de Boo TM, Merkus HM, Steegers EA. Longitudinal vitamin and homocysteine levels in normal pregnancy. Br J Nutr 2001;85:49-58.

14. De la Calle M, Usandizaga R, Sancha M, Magdaleno F, Cabrillo E. Homocisteína, ácido fólico y vitaminas del grupo B en ginecología y obstetricia. Actualidad Obstet Ginecol 2001;13:237-48.

15. Menéndez A, Britto JE. Metabolismo de la homocisteína y su relación con la aterosclerosis. Rev Cubana Invest Biomed 1999;18:155-68.

16. Sharma P, Senthilkumar R, Brahmachari V, Sundaramoorthy E, Mahajan A, Sharma A, et al. Mining literature for a comprehensive pathway analysis: a case study for retrieval of homocysteine related genes for genetic and epigenetic studies. Lipids Health Dis 2006;5:1.

17. Forges T, Monnier P, Alberto JM, Guéant RM, Daval JL, Gueant JL. Impact of folate and homocysteine metabolism on human reproductive health. Hum Reprod Update 2007;13:225-38.

18. Öztürk Ö, Karaer S, Dilara U, Efesoy A. Serum Homocysteine, Folate, and Vitamin B12 Levels in Pregnant and Non-Pregnant Women. Turkiye Klinikleri J Med Sci 2006;26:121-5.

19. Andersson A, Hultberg B, Brattstrom L, Isaksson A. Decreased serum homocysteine in pregnancy. Eur J Clin Chem Clin Biochem 1992;30:377-9.

20. Milman N, Byg KE, Hvas AM, Bergholt T, Eriksen L. Erythrocyte folate, plasma folate and plasma homocysteine during normal pregnancy and postpartum: a longitudinal study comprising 404 Danish women. Eur J Haematol 2006;76:200-5. 
21. Guerra EM, Morita OE, Peres S, Pagliusi RA, Sampaio LF, D'Almeida V, et al. Low ratio of S-adenosylmethionine to $\mathrm{S}$-adenosylhomocysteine is associated with vitamin deficiency in Brazilian pregnant women and newborns. Am J Clin Nutr 2004;80:1312-21.

22. Hague WM. Homocysteine and pregnancy. Best Pract Res Clin Obstet Gynaecol 2003;17:459-69.

23. Walker MC, Smith GN, Perkins SL, Keely EJ, Garner PR. Changes in homocysteine levels during normal pregnancy. Am J Obstet Gynecol 1999;180:660-4.

24. Montoya NE, Correa JC. Curvas de peso al nacer. Rev Salud Pública 2007;9:1-10.

25. Babson AL, Olson DR, Palmieri T, Ross AF, Becker DM, Mulqueen PJ. The Immulite assay tube: a new approach to heterogeneous ligand assay. Clin Chem 1991;37:1521-2.

26. Shipchandler MT, Moore EG. Rapid, fully automated measurement of plasma homocyst (e) ine with the Abbott IMx analyzer. Clin Chem 1995;41:991-4.

27. Maniatis T, Fristsch E, Sambrook J. Molecular cloning: a laboratory manual. Cold Spring Harbor, New York: Cold Spring Harbor Laboratory; 1982.

28. Bermúdez M, Briceño I, Gil F, Bernal J. Homocisteína y polimorfismos de cistationina $\beta$ sintasa y metilentetrahidrofolato reductasa en población sana de Colombia. Colomb Med 2006;37:46-52.

29. van der Put NMJ, Blom HJ. Reply to Donnelly. Am J Hum Genet 2000;66:744-5.

30. Ananth CV, Elsasser DA, Kinzler WL, Peltier MR, Getahun D, Leclerc D, et al. Polymorphisms in methionine synthase reductase and betainehomocysteine S-methyltransferase genes: Risk of placental abruption. Mol Genet Metab 2007;91: 104-10.

31. Lievers KJ, Kluijtmans LA, Heil SG, Boers GH, Verhoef P, van Oppenraay-Emmerzaal D, et al. A 31 bp VNTR in the cystathionine beta-synthase (CBS) gene is associated with reduced CBS activity and elevated post-load homocysteine levels. Eur J Hum Genet 2001;9:583-9.

32. Afman LA, Lievers KJ, Kluijtmans LA, Trijbels FJ, Blom HJ. Gene-gene interaction between the cystathionine beta-synthase 31 base pair variable number of tandem repeats and the methylenetetrahydrofolate reductase 677C > T polymorphism on homocysteine levels and risk for neural tube defects. Mol Genet Metab 2003;78:211-5.

33. Gan YY, Chen CF. Novel alleles of 31-bp VNTR polymorphism in the human cystathionine $\beta$-synthase (CBS) gene were detected in healthy Asians. J Genet 2010;89:449-55.

34. Lievers KJA, Kluijtmans LAJ, Blom HJ, Wilson PW, Selhub J, Ordovas JM. Association of a $31 \mathrm{bp}$ VNTR in the CBS gene with postload homocysteine concentrations in the Framingham Offspring Study. Eur J Hum Genet 2006;14:1125-9.

35. Rozen S, Skaletsky H. Primer3 on the WWW for general users and for biologist programmers. Methods Mol Biol 2000;132:365-86.

36. Roberts RJ, Vincze T, Posfai J, Macelis D. REBASEa database for DNA restriction and modification: enzymes, genes and genomes. Nucleic Acids Res 2010;38:D234-D6.

37. López E, Vilaseca MA, Lailla JM. Plasma total homocysteine in uncomplicated pregnancy and in preeclampsia. Eur J Obstet Gynecol Reprod Biol 2003;108:45-9.

38. González Gross M, Sola R, Castillo M. Folato: una vitamina en constante evolución. Med Clin 2002;119:627-35.

39. Stabler SP, Allen RH. Vitamin B12 deficiency as a worldwide problem. Annu Rev Nutr 2004;24: 299-326.

40. Aubard Y, Darodes N, Cantaloube M. Hyperhomocysteinemia and pregnancy-review of our present understanding and therapeutic implications. Eur J Obstet Gynecol Reprod Biol 2000;93:157-65.

41. Murphy MM, Scott JM, McPartlin JM, FernandezBallart JD. The pregnancy-related decrease in fasting plasma homocysteine is not explained by folic acid supplementation, hemodilution, or a decrease in albumin in a longitudinal study. Am J Clin Nutr 2002;76:614-9.

42. Bonnette RE, Caudill MA, Boddie AM, Hutson AD, Kauwell GP, Bailey LB. Plasma homocyst(e)ine concentrations in pregnant and nonpregnant women 
with controlled folate intake 2. Obstet Gynecol 1998;92:167-70.

43. Patrick TE, Powers RW, Daftary AR, Ness RB, Roberts JM. Homocysteine and folic acid are inversely related in black women with preeclampsia. Hypertension 2004;43:1279-82.

44. Sanchez SE, Zhang C, Rene Malinow M, WareJauregui S, Larrabure G, Williams MA. Plasma folate, vitamin B12, and homocyst (e) ine concentrations in preeclamptic and normotensive Peruvian women. Am J Epidemiol 2001;153:474-80.

45. Vollset SE, Refsum H, Irgens LM, Emblem BM, Tverdal A, Gjessing HK, et al. Plasma total homocysteine, pregnancy complications, and adverse pregnancy outcomes: the Hordaland Homocysteine Study. Am J Clin Nutr 2000;71:962-8.

46. Cardona H, Cardona-Maya W, Gómez JG, Castañeda S, Gómez JM, Bedoya G, et al. Relationship between methylenetetrahydrofolate reductase polymorphism and homocysteine levels in women with recurrent pregnancy loss: a nutrigenetic perspective. Nutr Hosp 2008;23:277-82.

47. Tamura T, Picciano MF. Folate and human reproduction. Am J Clin Nutr 2006;83:993-1016.

48. Chávez DV, Velazco MR, Sanin LH, Levario M, Aguirre AA, Martínez LE. Relation Between Levls of Folic acid, Vitamin B12 and Maternal Homocysteine with Neural Tube Defects and Cleft Lip. Int J Morphol 2008;26:905-14.

49. López JS, Castilla EE, Orioli IM. Folic acid flour fortification: Impact on the frequencies of 52 congenital anomaly types in three South American countries. Am J Med Genet A 2010;152A:2444-58.

50. US Food and Drug Administration. Food standards: amendment of standards of identity for enriched grain products to require addition of folic acid. Fed Regist 1996;61:8781-97.
51. Hertrampf E, Cortés F. Folic acid fortification of wheat flour: Chile. Nutr Rev 2004;62:S44-S8.

52. Schulpis K, Spiropoulos A, Gavrili S, Karikas G, Grigori C, Vlachos G, et al. Maternal-neonatal folate and vitamin B12 serum concentrations in Greeks and in Albanian immigrants. J Hum Nutr Diet 2004;17:443-8.

53. Garcia-Casal M, Osorio C, Landaeta M, Leets I, Matus P, Fazzino F, et al. High prevalence of folic acid and vitamin B12 deficiencies in infants, children, adolescents and pregnant women in Venezuela. Eur J Clin Nutr 2005;59:1064-70.

54. Forrellat M, Góms I, Gautier du Défaix H. Vitamina B12: metabolismo y aspectos clínicos de su deficiencia. Rev Cubana Hematol Inmunol Hemoter 1999;15: 159-74.

55. Green R. Is it time for vitamin B-12 fortification? What are the questions? Am J Clin Nutr 2009;89:712S-6S.

56. Carmel R. Mandatory fortification of the food supply with cobalamin: an idea whose time has not yet come. J Inherit Metab Dis 2011;34:67-73.

57. Ghezzi F, Tibiletti MG, Raio L, Di Naro E, Lischetti B, Taborelli M, et al. Idiopathic fetal intrauterine growth restriction: a possible inheritance pattern. Prenat Diagn 2003;23:259-64.

58. Ruoti M, Loú LA, Fabré E. Papel de homocisteína en el metabolismo celular y su relación con el embarazo. Progresos en diagnóstico y tratamiento prenatal 2003;15:32-4.

59. D’Uva M, Di Micco P, Strina I, Alviggi C, Iannuzzo M, Ranieri A, et al. Hyperhomocysteinemia in women with unexplained sterility or recurrent early pregnancy loss from Southern Italy: a preliminary report. Thromb J 2007;5:10.

60. Engel SM, Olshan AF, Siega-Riz AM, Savitz DA, Chanock SJ. Polymorphisms in folate metabolizing genes and risk for spontaneous preterm and smallfor-gestational age birth. Am J Obstet Gynecol 2006;195:1231.e1-e11. 